\title{
AÇÕES DO CENTRO DE ATENÇÃO PSICOSSOCIAL PARA A REABILITAÇÃO DO PORTADOR DE SOFRIMENTO PSÍQUICO*
}

Jacira Alves das Neves ${ }^{1}$, Priscilla Maria de Castro Silva², Elisângela Braga de Azevedo ${ }^{3}$, Juliana de Oliveira Musse ${ }^{4}$, Maria de Oliveira Ferreira Filha ${ }^{5}$

RESUMO: Objetivou-se com este estudo investigar as ações de saúde mental destinada à reabilitação psicossocial do portador de sofrimento psíquico em Centro de Atenção Psicossocial (CAPS) e envolveu nove usuários, cinco profissionais e seis familiares. Trata-se de um estudo de caráter qualitativo, exploratório e descritivo, realizado no período de março a maio de 2009. O material empírico sofreu análise do tipo categorial-temática, proposta por Bardin, e desta análise extraíram-se as categorias O processo de trabalho do CAPS na concepção dos profissionais do serviço; A concepção do usuário no processo de reabilitação psicossocial; e A participação da família na ressocialização do portador de sofrimento psíquico. A pesquisa confirma o CAPS como um dispositivo significativo no processo de reabilitação do portador de sofrimento psíquico, corroborando as expectativas da Reforma Psiquiátrica, entretanto, este processo exige aprimoramento das ações desenvolvidas pelo profissional e maior envolvimento da família para uma efetiva reabilitação psicossocial. PALAVRAS-CHAVE: Reabilitação; Reforma psiquiátrica; Saúde mental.

\section{ACTIONS IN A PSYCHO-SOCIAL CARE CENTER FOR THE REHABILITATION OF PERSONS WITH PSYCHOLOGICAL DISORDERS}

ABSTRACT: The purpose of this study was to investigate mental health actions aimed at psychosocial rehabilitation of persons with mental disorders in a psycho-social care center(PSCC), and involved nine service users, five professionals, and six family members. It is an exploratory, descriptive study with a qualitative character, carried out between March and May of 2009. The empirical material underwent the thematic categorical analysis proposed by Bardin, which resulted in the following categories: The work process of the PSCC in the conception of professionals in the work; The conception of the service user undergoing psychosocial rehabilitation; and The participation of the family in the re-socialization of the person with mental disorder. The research confirmed the PSCC as a significant tool in the rehabilitation process of persons with mental disorders, corroborating the expectations of the Psychiatric Reform, however, for effective psychosocial rehabilitation, this process demands both improvement of the actions carried out by health professionals and greater involvement of the family.

KEYWORDS: Rehabilitation; Psychiatric reform; Mental health.

\section{ACCIONES DEL CENTRO DE ATENCIÓN PSICOSOCIAL PARA REHABILITACIÓN DEL PORTADOR DE SUFRIMIENTO PSÍQUICO}

RESUMEN: Este estudio tuvo el objetivo de investigar las acciones de salud mental destinadas a la rehabilitación psicosocial del portador de sufrimiento psíquico en Centro de Atención Psicosocial e involucró nueve usuarios, cinco profesionales y seis familiares. Este es un estudio de carácter cualitativo, exploratorio y descriptivo, realizado en el periodo de marzo a mayo de 2009. El material empírico pasó por análisis del tipo categorial y temático, propuesto por Bardin, y de ese análisis surgieron las categorías El proceso de trabajo de CAPS en la concepción de los profesionales del servicio; La concepción del usuario en el proceso de rehabilitación psicosocial; y La participación de la familia en la resocialización del portador de sufrimiento psíquico. La investigación confirma el CAPS como un dispositivo significativo en el proceso de rehabilitación del portador de sufrimiento psíquico, corroborando las expectativas de la Reforma Psiquiátrica, sin embargo, este proceso exige perfeccionamiento de las acciones desarrolladas por el profesional y mayor participación de la familia para una efectiva rehabilitación psicosocial.

PALABRAS CLAVE: Rehabilitación; Reforma psiquiátrica; Salud mental.

*Este artigo é um recorte do Trabalho de Conclusão de Curso da Graduação em Enfermagem na Faculdade de Ciências Médicas de Campina Grande - PB intitulado "A importância do Centro de Atenção Psicossocial (CAPS) de Araruna na ressocialização do usuário com transtorno mental".

${ }^{1}$ Enfermeira. Membro do Grupo de Estudos e Pesquisas em Saúde Mental Comunitária da Universidade Federal da Paraíba - UFPB.

${ }^{2}$ Enfermeira. Mestranda pelo Programa de Pós-Graduação em Enfermagem da UFPB. Professora do Departamento de Enfermagem da Universidade Federal de Campina Grande - PB. Membro do Grupo de Estudos e Pesquisas em Saúde Mental Comunitária.

${ }^{3}$ Enfermeira. Doutoranda em Enfermagem pelo Programa de Pós-Graduação da UFPB. Professora do Departamento de Enfermagem Faculdade de Ciências Médicas. Membro do Grupo de Estudos em Saúde Mental Comunitária.

${ }^{4}$ Enfermeira. Mestranda em SaúdePúblicapelaUniversidadeEstadual da Paraíba. Membro do Grupo deEstudose Pesquisas em SaúdeMental Comunitária. ${ }^{5}$ Enfermeira. Doutora em Enfermagem. Professora do Departamento e Programa de Pós-Graduação em Enfermagem da UFPB. Líder do Grupo de Estudos e Pesquisas em Saúde Mental Comunitária.

Autor correspondente:

Recebido: 25/11/2011

Juliana de Oliveira Musse Aprovado: 04/06/2012

Universidade Federal da Paraíba

Rua Alvino de Farias Pimentel, 36 - 58411010-Campina Grande-PB-Brasil

E-mail: julimusse@hotmail.com 


\section{INTRODUÇÃO}

No dia 6 de abril de 2001 foi promulgada no Brasil a Lei 10.216 que dispõe sobre a proteção e os direitos dos portadores de sofrimento psíquico, redirecionando o modelo assistencial em saúde mental ${ }^{(1)}$.

Com a Reforma Psiquiátrica, os direitos das pessoas com sofrimento psíquico passaram a ser mais respeitados, agora protegidas por Lei, e passaram a ter um tratamento mais humanizado, assegurado e protegido contra qualquer forma de exclusão e maus tratos, desconstruindo gradativamente a lógica manicomial. Neste novo modelo de atenção em saúde mental prevalece o tratamento em serviço de base comunitária, tendo como dispositivo mais atuante e efetivo o Centro de Atenção Psicossocial (CAPS) ${ }^{(2)}$.

O CAPS tem como função prestar assistência holística em regime de atenção diária, evitando as internações em hospitais psiquiátricos; regular a porta de entrada da rede de cuidado em saúde mental na sua área de atuação; dar suporte e supervisionar a atenção à saúde mental na rede básica e promover reabilitação psicossocial das pessoas com sofrimento psíquico através de ações intersetoriais ${ }^{(3)}$.

Partindo da perspectiva da reabilitação psicossocial do portador de sofrimento psíquico, a família age como alicerce, pois ela transcende qualquer esfera política, social ou qualquer outro gênero de agrupamento humano ${ }^{(4)}$. Os transtornos psíquicos podem eclodir a partir dos conflitos existentes nas relações familiares sendo, portanto, de grande relevância o envolvimento da família para efetivação do tratamento.

Levando em conta o papel e relevância do CAPS, é necessário enfatizar o acompanhamento dos atores envolvidos nesse processo de mudança, baseado agora na comunidade e na realidade em que o sujeito está inserido.

Logo, este trabalho objetivou investigar as ações de saúde mental destinada à reabilitação psicossocial do portador de sofrimento psíquico do CAPS, incluindo: caracterizar as atividades desenvolvidas pela equipe de trabalho do CAPS no tocante a reabilitação psicossocial; verificar as concepções e importância da família no processo de ressocialização do portador de sofrimento psíquico após o advento do CAPS no município; analisar se com o advento da Reforma Psiquiátrica e do CAPS, houve melhoria da qualidade de vida e, consequente, redução da discriminação ao portador de sofrimento psíquico.

\section{MÉTODO}

Trata-se de uma pesquisa do tipo exploratória e descritiva com abordagem qualitativa. Foi realizada no período de março a abril de 2009, no CAPS I de Araruna, Paraíba. Para coleta do material empírico utilizou-se um roteiro de entrevista semiestruturado na qual foram direcionados questionamentos aos usuários, familiares e profissionais do CAPS.

Uma das questões norteadoras utilizadas para os três grupos pesquisados foi: Fale-me sobre a importância do CAPS para a concretização da reabilitação psicossocial do portador de sofrimento psíquico? Para os profissionais perguntou-se: Quais as atividades que são desenvolvidas no CAPS que favorecem a ressocialização e a reabilitação psicossocial? Para os familiares indagou-se: Qual a importância da família no processo de ressocialização e reabilitação psicossocial? E para os usuários, além de se perguntar quais atividades eles mais se identificavam, questionou-se também, se houve melhorias na qualidade de vida do usuário após sua inserção no CAPS, e quais atividades eles gostariam que fossem acrescentadas no serviço, para uma melhor evolução em seu tratamento.

Para coleta do material empírico foram envolvidos cinco profissionais que trabalhavam efetivamente no CAPS, uma pedagoga, uma enfermeira, uma assistente social, um psicólogo e um cuidador, todos participaram da pesquisa por acessibilidade e se enquadraram no critério de inclusão, que foi trabalhar há mais de seis meses no serviço. Seis familiares de usuários que eram atendidos no CAPS foram convidados, e como critério de inclusão delimitou-se participar assiduamente do grupo de família desenvolvido no CAPS e ser maior de 18 anos de idade. Por fim, 9 usuários deste serviço também participaram da pesquisa, que por sua vez, tiveram como critérios de inclusão estar em tratamento há mais de seis meses e participar de alguma oficina destinada à Reabilitação Psicossocial em desenvolvimento no serviço, e ser maior de 18 anos.

As entrevistas foram gravadas em um aparelho de Mídia Player (Mp4), assim, realizou-se sua transcrição em literalidade, leu-se exaustivamente e categorizou-se o conteúdo de acordo com suas afinidades e pertinência ${ }^{(5)}$. Os sujeitos foram identificados pelas letras U (para usuário), F (para familiar) e $\mathrm{P}$ (para profissionais), respectivamente, seguido de um numeral sequencial.

Foram considerados os aspectos éticos da pes- 
quisa, envolvendo seres humanos recomendados pela resolução 196/96 do Conselho Nacional de Saúde ${ }^{(6)}$. O projeto de pesquisa foi aprovado pelo Comitê de Ética em Pesquisa da UEPB, através do número de CAAE0211.0.133.000-08. Todos os participantes assinaram o Termo de Consentimento Livre e Esclarecido.

\section{RESULTADOS}

Para um melhor entendimento do material empírico criou-se três categorias: $O$ processo de trabalho do CAPS na concepção dos profissionais do serviço; A concepção do portador de sofrimento psíquico no processo de reabilitação psicossocial; A participação da família na ressocialização do usuário.

\section{O processo de trabalho do CAPS na concepção dos profissionais do serviço}

Nessa categoria analisaram-se as concepções dos profissionais que atuam no serviço, a relevância deste para a melhoria da qualidade de vida do usuário que o frequenta e a consequente reabilitação psicossocial. Com relação às atividades desenvolvidas no CAPS, pode-se perceber grande diversidade de ações por parte dos profissionais:

O CAPS tem todo um aparato de atividades direcionado de acordo com a atividade de cada profissional, envolve desde atividades lúdicas a atendimento psiquiátrico [...] oficinas pedagógicas que desenvolvem atividades relacionadas à alfabetização, desenvolvimento da condenação motora daqueles usuários que tem uma dificuldade maior muitas vezes até pela impregnação da medicação [...]. Além da oficina pedagógica, tem a oficina de teatro, a oficina de bijuterias, trabalhos manuais. Tem uma diversidade de atividades onde ele se insere de acordo com habilidade de cada um. (P.1)

No CAPS nós dispomos de um quadro de psicólogos, médicos, enfermeiros, oficineiros, etc. Nós fazemos aula de teatro, oficina pedagógica, oficina com filmes vídeos. Na terça-feira é o dia da beleza onde nós cuidamos dos cabelos das unhas da higiene pessoal [...]. Na quarta-feira é dia da oficina de artesanato, trabalhos manuais. Na sexta trabalhamos a questão da evangelização para todas as pessoas, não abordamos temas polêmicos, mas tema que venha servir de lição para a vida de cada um. (P.2)
Acerca da reabilitação psicossocial do usuário, os profissionais afirmaram que as atividades desenvolvidas pelo serviço buscam a valorização da pessoa humana:

Essas atividades servem para valorizar o usuário, e promover essa reinserção a exemplo da oficina de teatro [...]. Tem o objetivo de promover a inserção social deste usuário e a integração ao convivio social. (P.1)

As atividades feitas aqui geralmente são feitas em comunhão com a comunidade, fizemos a paixão de Cristo, os atores do CAPS junto com o grupo Serra de teatro que já tem vinte anos de teatro. Foi uma coisa tão produtiva porque ninguém conseguia perceber quem era do CAPS e quem não era do CAPS [...]. Tudo que na comunidade existe o pessoal do CAPS está sempre participando de forma ativa. (P.2)

A valorização quanto pessoa. Essas pessoas são ditas loucas e totalmente desvalorizadas, a gente busca mostrar para sociedade que não é assim. (P.3)

Em relação à concepção dos profissionais sobre a assimilação das atividades pelo usuário eles responderam:

[...] é impressionante, eles conseguem se adaptar, eles conseguem aprender e assim a gente descobre que cada um tem um dom, prá pintura, prá dança, prá artes manuais; o teatro então cada um dentro da sua capacidade. (P.3)

É gradual, o usuário deve passar por várias oficinas chamadas de porta de entrada, assim ele entra no serviço e não conhecemos a habilidade que ele tem, nem a afinidade com algumas atividades. Então, ele passará por todas as oficinas e ai é descoberto onde ele se encaixa melhor, depois oferece essa oficina a ele. (P. 4)

Nós podemos dizer que setenta por cento de assimilação, porque tem algumas pessoas que têm alguma resistência em participar. (P.2)

\section{A concepção do portador de sofrimento psíquico no processo de reabilitação psicossocial}

Nessa categoria avaliou-se o que os atores diretos desse processo de reabilitação psicossocial, relataram sobre a importância do CAPS em suas vidas. Os usuários do CAPS relataram o que mais gostam de fazer: 
Eu gosto de escrever texto sobre o que está acontecendo no Brasil e no mundo [...] escrevo sobre o carnaval e, quando é época de São João, escrevo sobre o São João e assim por diante. (U.1)

Gosto de fazer todos os trabalhos que tem aqui, principalmente fazer florzinha. (U.2)

Gosto de pintar, escrever, desenhar, dançar, das aulas de teatro. Eu gosto de tudo aqui! (U.3)

Quando indagados sobre a melhora em sua vida após sua inserção nos CAPS, os usuários referiram mudanças em diferentes aspectos, como novas interações interpessoais, a melhoria da autoestima e menor recorrência de crises que diminuíram as internações hospitalares:

Melhorou muito porque eu só ficava em casa, solitário. Às vezes, assistindo televisão ou escutando o rádio e não tinha muito significado e aqui melhorou porque a gente conhece gente diferente. (U.1)

Melhorou muita coisa. Se não fosse o CAPS eu já tinha morrido [...]. Eu não tomava banho, agora eu consigo, dentro do CAPS, eu tomo banho, escovo meus dentes que antes não escovava [...]. (U.4)

A doença melhorou muito, hoje eu tô outra à vista do que eu era. Fui internada cinco vezes em João Pessoa, depois que eu vim prá aqui não fui mais, quer dizer que eu tô bastante melhor. (U.3)

Os usuários relataram uma frequente participação nas atividades desenvolvidas pelo CAPS, como percebe-se nos depoimentos abaixo:

Participo, faço oficinas, brincadeiras, tem pinturas, tem várias coisas. (U.6)

Sim, participo frequentemente. Todos os dias! (U.3)

Participo sempre. Eu venho duas vezes por semana participo de oficina de artesanato, participo das aulas de pedagogia e muitas outras obrigações. (U.1)

Participo sim. Eu faço tarefinha de escola, eu faço tudo aqui, converso com as minhas amigas que eu gosto, com as que eu não gosto também não converso. (U.5)

Participo todo dia. Eu era bem magrinha quando eu vim prá aqui, agora eu estou gorda. (U.4)

Nos discursos dos usuários quando indagados sobre as atividades que eles gostariam que fossem realizadas no CAPS, alguns demonstraram interesse, principalmente, pela aula de pedagogia e oficina de pintura:

Por enquanto está bom, têm algumas atividades que é pouco tempo, como a aula de pedagogia. Ela tem pouco tempo pra dar aula prá gente, já a de teatro, ela dá bastante. Queria que aumentasse o tempo da pedagoga. (U.1)

[...] a pintura sabe eu sou doidinha. Voltar a trabalhar aqui [...].(U.3)

\section{A participação da família na ressocialização do portador de sofrimento psíquico}

Nessa categoria foi proposta a análise das concepções dos familiares acerca das principais mudanças ocorridas na vida do portador de sofrimento psíquico e a sua importância no processo de reabilitação psicossocial.

Os familiares, quando questionados sobre a melhora de seu ente após frequentar o CAPS, relataram que o tratamento fez com que a convivência com usuário se torna-se mais pacífica, no momento em este se encontra mais calmo, menos violento e as crises se tornam menos frequentes:

[...] ele só vivia aperreando dentro de casa. Depois que eu comecei vim prá aqui, ele começou tomar o remédio controlado. Agora tá mais calmo, vive bem melhor. (F.1)

[...] senti que ele tá melhor, ele não dormia muito, agora tá dormindo que faz gosto! Depois que tá tomando esses comprimidos, dorme a noite todinha, acorda de manhã. Prá mim é uma beleza [...]. (F.3).

Ela ficou mais calma, ela tá mais à vontade. Aqui, acolá, dá a crise dela, mas em casa ela não tem violência comigo, está bem melhor. (F.2)

Em relação ao trabalho desenvolvido no CAPS e a ajuda no processo de ressocialização, os familiares referiram um grande progresso com a intervenção desse serviço:

O processo é muito bom, todo trabalho vem sendo muito aproveitado, éuma coisa excelente, éuma maravilha. (F.4) 
Tem ajudado e se não fosse aqui pior seria pra ele. Mas, como a gente tá aqui está servindo demais, ele vivia sofrido, agora [...] ele é bem recebido, tô achando muito bom. (F. 3)

[...] mas agora a gente se abre aqui no CAPS. A gente se desenvolveu mais, e graças a Deus tem ajudado muito. (F. 1)

Sim, ela faz tudo, ela vai à feira, sempre vai à missa e cuida bem de casa também. (F.5)

Quando interrogados como é visto o sofrimento psíquico de seu familiar e se houve diminuição da discriminação, os mesmos afirmaram que já existe uma grande aceitação por parte de algumas pessoas, principalmente familiares, no entanto, a discriminação ainda se faz presente em alguns casos:

Uma parte discrimina, outra aceita [...]. Tem umas pessoas na familia que aceita, outras acham que aquilo ali é porque ela quer, mas não é, porque ninguém pede prá ter problemas. (F. 4)

De fora assim é discriminação [...]. Minha família todos são calmo com eles, não tem discriminação[...]. É tudo numa boa, então eles têm muita pena deles serem assim. (F. 4).

Com aceitação. Minha família todos gostam dele, os amigos dele gosta dele também. Quando ele chega, os amigos chega todos ao redor conversando com ele, onde ele chega é aquela festa que o povo faz com ele. (F.3)

\section{DISCUSSÃO}

Os achados desse estudo revelaram que as atividades desenvolvidas pelos profissionais no CAPS corroboram com o Ministério da Saúde, quando este explicita que é incumbência do serviço ofertar diversos tipos de atividades terapêuticas, não limitadas ao uso de consultas e de medicamentos. Devem disponibilizar diversos recursos terapêuticos, a exemplo de psicoterapias individuais e grupais, oficinas terapêuticas, atividades comunitárias, atividades artísticas, orientação e acompanhamento do uso de medicação e atendimento domiciliar, incluindo os familiares ${ }^{(1)}$.

Contextualizando os depoimentos dos profissionais acerca da reinserção social do portador de sofrimento psíquico, pesquisadores afirmam que o CAPS tem como filosofia rever as relações internas de poder, a inclusão social do portador de sofrimento psíquico, utilizar recursos da comunidade, envolver familiares no tratamento, trabalhando para a autonomia do sujeito. Nessa perspectiva, o papel dos profissionais e sua ação diária prevê mudança de postura e adaptação contínua às diferentes demandas e situações ${ }^{(7)}$.

No CAPS procuram-se meios de se aproximar os sujeitos do contexto e esses devem se inserir e dialogar com esse entorno, considerando-se parte significativa da dinâmica de cuidar. Nesse sentido, o portador de sofrimento psíquico apropria-se dos conhecimentos já produzidos, articula-os com sua observação/ação e, em conjunto, os profissionais, pacientes e familiares, produzem novos conhecimentos ${ }^{(8)}$.

Estudo realizado em 2011 revelou que os espaços encontrados no CAPS estão promovendo a interação entre a equipe e os usuários do serviço, isto em decorrência da iniciativa, tanto dos profissionais como dos usuários, em construir de forma coletiva o projeto terapêutico e as reuniões de equipe ${ }^{(9)}$. Sendo esse um fator impulsionador para a efetivação da integralidade do cuidado, tão almejada pelo sistema de saúde vigente no país.

Contextualizando sobre os tipos de atividades que os usuários mais gostam de realizar no CAPS, as terapias grupais têm se revelado como uma atividade cada vez mais importante no processo de reabilitação e inclusão social. Inúmeros fatores contribuem para a crescente ênfase no trabalho grupal como, por exemplo, a proposta da interdisciplinaridade em saúde; a racionalização do tempo de execução das atividades, as quais não poderiam ser feitas individualmente com cada cliente; o resgate da socialização dos pacientes e o combate ao individualismo ${ }^{(10)}$.

Os depoimentos dos usuários refletem melhorias em suas vidas após a inserção no CAPS, o que demonstra que esse serviço, como dispositivo na rede de saúde mental, tem assegurado melhora além do quadro psíquico, como em outras áreas concernente à vida do portador de sofrimento psíquico.

Os relatos explicitaram a frequente participação do usuário nas atividades desenvolvidas, o que vem demonstrar a eficácia da proposta desse serviço durante o processo de inclusão social, no momento em que as pessoas em sofrimento psíquico aderem às atividades em seu cotidiano. O trabalho vivo ocorre no momento singular do encontro dos sujeitos, ou seja, é o momento da criação, exercido de forma autônoma; essa dinâmica de trabalho encontrada nos CAPS é também representadas através das oficinas terapêuticas ${ }^{(11)}$. 
Quando indagados sobre as atividades que gostariam de participar no CAPS, os usuários referiram a aula de pedagogia e oficina de pintura, isso vem acrescentar o quanto as atividades terapêuticas expressivas são importantes no processo do cuidar. A arte pode ser compreendida, no campo da saúde mental, como um recurso para a humanização dos cuidados em saúde. No CAPS ela é utilizada nas atividades como produtora de subjetividade, catalisadora de afetos, engendradora de territórios e meio de produção e inserção social das pessoas portadoras de sofrimento psíquico ${ }^{(12)}$.

Desse modo, percebe-se que a família tem papel fundamental para o sucesso do tratamento do sujeito. Contudo, este envolvimento é um processo recente, se considerarmos que, há cerca de uma década, o único modelo de atendimento era o manicômio. Ainda que se considere a participação familiar como fundamental para o tratamento de seus usuários e que este discurso seja frequentemente reproduzido, tal participação restringe-se às reuniões/grupos de família que discutem assuntos relativos à medicação e a como lidar com alguns comportamentos inerentes à doença.

De acordo com os familiares, houve progresso no comportamento do seu ente após o tratamento oferecido pelo CAPS, no que diz respeito à convivência, à frequência das crises psicóticas e, até mesmo, aos episódios de violência. A percepção dos sujeitos quanto ao cuidado e ao tratamento dos portadores de doença mental se reflete, principalmente, na medicação. A mesma trata e previne a fase aguda da doença. Ao reduzir os sintomas, os fármacos e procedimentos recomendados pelo médico possibilitam a introdução de outras formas de tratamento e organiza o mundo interno do paciente, o que facilita sua reintegração à realidade externa ${ }^{(13)}$.

Em relação ao trabalho desenvolvido no CAPS e a ajuda no processo de reabilitação psicossocial, os familiares referiram grandes avanços no tratamento após a intervenção desse serviço. Nesse contexto, esse serviço substitutivo busca não apenas a desinstitucionalização da loucura, mas o combate ao isolamento e à exclusão do usuário da vida em sociedade. Assim, faz-se necessário um processo de desmontagem dos saberes que comprimem essa experiência da loucura no interior da sintomatologia da doença mental, com concomitante criação de novos modos de sociabilidade e produção de valor social ${ }^{(14)}$.

O familiar cuidador admite a discriminação do usuário em grau menor junto à família e maior extra família, embora reconheça que a aceitação é maior que a discriminação, ainda que considerem mínima a participação do usuário no cotidiano extra CAPS. A estigmatização e a discriminação ligadas aos transtornos mentais estão fortemente associadas com o sofrimento, as incapacidades e as perdas econômicas ${ }^{(15)}$.

Alguns familiares expressaram o alto grau de dependência do ente em sofrimento psíquico, dessa forma, é necessário salientar que a família é um suporte básico para a vida de qualquer pessoa. Mas, para os doentes mentais psicóticos ela possui importância, pelo fato desses sujeitos, na maioria das vezes, necessitarem de cuidados e acompanhamento dos membros do grupo familiar ${ }^{(16)}$.

Sabe-se que a reabilitação psicossocial configurase como um conjunto de meios, programas e serviços, que se desenvolvem para facilitar a vida do portador de sofrimento psíquico. Entretanto, os entraves como questões que envolvem a discriminação e a concepção de que o portador de sofrimento psíquico é um ser improdutivo, nos permitem compreender a sutileza relacionada com a mudança das práticas coletivas e das concepções atuais da Reforma Psiquiátrica. Esta quebra de preconceito certamente não se fará somente com mudanças políticas, mas demandam um processo lento e gradativo de se repensar e modificar os paradigmas que direcionam as práticas de cuidado e as formas de reflexão da sociedade ${ }^{(17)}$.

\section{CONSIDERAÇÕES FINAIS}

Constatou-se que esse serviço de saúde assegura, através de seus profissionais, uma gama de recursos terapêuticos, os quais promovem uma prática cotidiana capaz de superar o isolamento do portador de sofrimento psíquico, envolvendo o familiar cuidador, via ações praticadas de acordo com a capacidade assimilativa de cada usuário e a habilidade em lidar com a diferença por parte de cada profissional.

Através dessa pesquisa identificou-se ainda, que os usuários participam efetivamente das terapias grupais e apontam uma melhora acentuada em sua condição de vida, principalmente com relação à sociabilidade, a partir do incremento da arte no processo de cuidar, que humaniza e contribui para a melhora do quadro geral de saúde. A família está inclusa nesse processo, efetivamente atua como facilitadora na condução do portador de transtorno mental. A maioria dos familiares reconhece o quadro evolutivo do portador após sua inserção no CAPS. 
OCAPS, como serviço substitutivo, tem papel preponderante em um novo conceito de ressocialização com resultados expressivos nesse âmbito. Contudo, é a renovação cotidiana dos atores envolvidos - profissionais, familiares e usuários - que potencializará novas conquistas.

\section{REFERÊNCIAS}

1. Ministério da Saúde (BR). Secretaria de Atenção à Saúde Departamento de Ações Programáticas Estratégicas. Saúde Mental no SUS: os Centros de Atenção Psicossocial. Brasília (DF); 2004

2. Fernandes A. Curso de especialização em saúde mental e atenção psicossocial. uma abordagem multidisciplinar. Fundação Francisco Mascarenhas; 2007.

3. Ministério da Saúde (BR) . Secretaria de Atenção à Saúde. DAPE. Coordenação Geral de Saúde Mental. Reforma psiquiátrica e política de saúde mental no Brasil. Documento apresentado à Conferência Regional de Reforma dos Serviços de Saúde Mental: 15 anos depois de Caracas. OPAS. Brasília; 2005.

4. Nogueira RCO. Que é ser família? há momentos em que somos de fato família e, em outros, somos qualquer coisa menos uma família. Jornal Missão Jovem. [Internet] 2009 [acesso em 23 mar 2009]. Disponível: http://www.pimenet.org.br/index.php?destino $=$ lista noticias \&menu=3\&nivel_1=49

5. BardinL. Análise de conteúdo. 20ª ed. Lisboa: Edições 70;2009.

6. Ministério da Saúde (BR). Resolução no 196/96 de 10 de outubro de 1996. Pesquisa envolvendo seres humanos. In: Informe epidemiológico do SUS. Brasília: Fundação Nacional de Saúde; 1996; (Suppl 3):278-91.

7. Onocko-Campos RT, Furtado JP. Entre a saúde coletiva e a saúde mental: um instrumental metodológico para avaliação da rede de Centros de Atenção Psicossocial (CAPS) do Sistema Único de Saúde. Cad. Saúde Pública. 2006;22(5):1053-62.

8. Chianca T, Marino E, Schiesari L. Desenvolvendo a cultura de avaliação em organizações da sociedade civil. São Paulo: Global; 2001.

9. Pereira DB, Coimbra VCC, Kantorski LP, Oliveira MM, Soares MC, Schrader GA integralidade no cotidiano das práticas em um Centro de Atenção Psicossocial. Cogitare enferm. 2011;16(3). [acesso em 30 abr 2012] Disponível: http://ojs.c3sl.ufpr.br/ojs2/index.php/ cogitare/article/view/21165/16226
10. Cavalcante TF, Guedes NG, Moreira RP, Guedes TG, Araújo MAM. Nursing care at new mental health services - experience report. Online braz. j. nurs. [Internet] 2006;5(1):23-9. [acesso em 30 abr 2012]. Disponível: http://www.objnursing.uff.br/index.php/ nursing/article/view/93

11. Coimbra VCC, Kantorski LP. O acolhimento em Centro de Atenção Psicossocial. Rev. enferm. UERJ. 2005;13(1):57-62.

12. Tavares CMM, Sobral VRS. Avaliação das praticas de cuidar envolvendo arte no âmbito do Centro de Atenção Psicossocial (CAPS). REME: rev. min. enferm. [Internet] 2005;9(2). [acesso em 19 mar 2009]. Disponível: http:// mail.live.com/default.aspx?wa=wsignin 1.0

13. Salles MM, Barros S. Reinternação em hospital psiquiátrico: a compreensão do processo saúde/doença na vivência do cotidiano. Rev Esc Enferm USP 2007; 41(1):73-81.

14. Silva MBB. Atenção psicossocial e gestão de populações: sobre os discursos e as práticas em torno da responsabilidade no campo da saúde mental. Physis. 2005;15(1):127-50.

15. Graham N; Lindesay J, Katona C, Bertolote JM, Camus V, Copeland JRM, et al. Redução da estigmatização e da discriminação das pessoas idosas com transtornos mentais: uma declaração técnica de consenso. Rev. psiq. clín. 2007;34(1):39-49.

16. Nasi C, Stumm LK, Hildebrandt LM. Convivendo com o doente psicótico na ótica do familiar. Rev. Eletr. Enf. [Internet]. 2004;06(1). [acesso em 2 abr 2009]. Disponível: http://www.fen.ufg.br/revista/revista6_1/ f6_mental.html

17. Santos JP, Souza MCBM, Oliveira NF. Reabilitação psicossocial na perspectiva de estudantes e enfermeiros da área de saúde mental Rev. Eletr. Enf. [Internet]. 2011;13(1). [acesso em 01 abr 2012]. Disponível: http:// www.fen.ufg.br/revista/v13/n1/v13n1a07.htm 